\title{
Band-selective dynamics in charge- transfer excited iron carbene complexes
}

\author{
Pavel Chábera, (D) a Lisa A. Fredin, (D) ${ }^{b}$ Kasper S. Kjær, (D) a \\ Nils W. Rosemann, (iD a Linnea Lindh, (DD a Om Prakash, ${ }^{c}$ Yizhu Liu, ${ }^{c}$ \\ Kenneth Wärnmark, ${ }^{c}$ Jens Uhlig, ${ }^{a}$ Villy Sundström, ${ }^{a}$ Arkady Yartsev ${ }^{a}$ \\ and Petter Persson (D) *b
}

\begin{abstract}
Received 7th December 2018, Accepted 18th February 2019
DOI: $10.1039 / c 8 f d 00232 k$
\end{abstract}

\begin{abstract}
Ultrafast dynamics of photoinduced charge transfer processes in light-harvesting systems based on Earth-abundant transition metal complexes are of current interest for the development of molecular devices for solar energy conversion applications. A combination of ultrafast spectroscopy and first principles quantum chemical calculations of a recently synthesized iron carbene complex is used to elucidate the ultrafast excited state evolution processes in these systems with particular emphasis on investigating the underlying reasons why these complexes show promise in terms of significantly extended lifetimes of charge transfer excited states. Together, our results challenge the traditional excited state landscape for iron-based light harvesting transition metal complexes through radically different ground and excited state properties in alternative oxidation states. This includes intriguing indications of rich band-selective excited state dynamics on ultrafast timescales that are interpreted in terms of excitation energy dependence for excitations into a manifold of chargetransfer states. Some implications of the observed excited state properties and photoinduced dynamics for the utilization of iron carbene complexes for solar energy conversion applications are finally discussed.
\end{abstract}

\section{Introduction}

Characterization of ultrafast excited-state dynamics is crucial for understanding and rationally improving the performance of a wide range of emerging solar energy conversion technologies, including molecular systems for both photovoltaic and solar fuels applications. ${ }^{1}$ Many molecular-based approaches utilize the favourable photoelectrochemical properties of transition metal complexes

\footnotetext{
${ }^{a}$ Division of Chemical Physics, Department of Chemistry, Lund University, Box 124, SE-22100 Lund, Sweden ${ }^{b}$ Division of Theoretical Chemistry, Department of Chemistry, Lund University, Box 124, SE-22100 Lund, Sweden.E-mail: Petter.Persson@teokem.lu.se

${ }^{c}$ Center for Analysis and Synthesis (CAS), Department of Chemistry, Lund University, Box 124, SE-22100 Lund, Sweden
} 
(TMCs) that have been developed both for light-harvesting and photocatalytic functions. ${ }^{2}$ TMCs can, in particular, utilize charge-transfer (CT) excited states as a first step towards generating high-energy CT states. The intrinsic capabilities of many transition metal complexes to sustain a range of oxidation states also make them suitable as central components for electrochemical and photoelectrochemical oxidation and reduction reactions as a key part of many photocatalytic reaction schemes. ${ }^{3}$

Octahedral second and third row transition metal complexes sporting $n \mathrm{~d}^{6}$ (with $n=4$ or 5 ) electronic configurations on the metal have maintained a central role for many light-harvesting and molecular photochemistry applications for several decades, owing in large part to the large ligand field splitting (LFS) in which the low-spin $n \mathrm{~d}^{6}$ electronic ground state configuration is stabilized. This has yielded many complexes with long-lived high-energy metal-to-ligand charge transfer (MLCT) excited states that have been widely used for photophysical and photochemical applications. ${ }^{2}$ This particularly includes the famous family of $\mathrm{Ru}^{\mathrm{II}}$ polypyridyl complexes such as $\mathrm{Ru}(\mathrm{bpy})_{3}$ derivatives used extensively as photosensitizers, e.g. in molecular photovoltaics. ${ }^{4,5}$ Other prominent examples of photoactive $\mathrm{d}^{6}$ complexes are based on the third-row species $\mathrm{Re}^{\mathrm{I}}, \mathrm{Os}^{\mathrm{II}}$, and $\mathrm{Pt}^{\mathrm{III}}$ which are also interesting for light-emitting applications. ${ }^{6}$

It has, however, increasingly been recognized that it is desirable for environmentally friendly and sustainable large-scale solar energy applications to replace rare and expensive second and third row transition metal complexes with more Earth-abundant alternatives. ${ }^{7-11}$ This has prompted significant interest in developing first row transition metal complexes with good photophysical and photochemical properties to ideally rival and replace those from the more wellestablished second and third row transition metals. This has led to the emergence in recent years of several interesting photoactive first row transition metal complexes of, for example, copper, chromium, and iron. ${ }^{12-16}$ As the lighter and abundant congener of ruthenium and osmium, iron looks prima facie to be an obvious candidate to develop photofunctional first row transition metal complexes. Progress in this direction has, however, until very recently been largely hampered by poor excited state properties, including a preponderance for substantial and ultrafast excited state energy losses, ${ }^{17,18}$ with high-energy metalto-ligand charge-transfer excited states in iron polypyridyl complexes typically decaying on a few picoseconds or faster timescale. ${ }^{\mathbf{1 9 2 0}}$ This has remained rather discouraging compared to the abundance of ruthenium polypyridyl complexes maintaining high excited state energies for hundreds of nanoseconds or longer. ${ }^{2}$

The photophysics and photochemistry of many first-row transition metal complexes, including traditional octahedral $\mathrm{Fe}^{\mathrm{II}}$ polypyridyl complexes, suffer severely from an intrinsically weaker ligand field splitting for $3 \mathrm{~d}$ complexes compared to their $4 \mathrm{~d}$ and $5 \mathrm{~d}$ counter-parts. ${ }^{21}$ Even the favourable $3 \mathrm{~d}^{6}$ configuration in octahedral $\mathrm{Fe}^{\mathrm{II}}$ complexes is typically not sufficient to avoid serious problems with ultrafast deactivation of high energy excited singlet and triplet ${ }^{1 /}$ ${ }^{3} \mathrm{MLCT}$ states to low-energy high-spin (quintet) metal centred $\left({ }^{5} \mathrm{MC}\right)$ scavenger states. ${ }^{22}$ Complexes with low-energy high-spin MC states are of some considerable interest in themselves as spin cross-over (SCO) complexes, and they are investigated in the particular context of photoinduced formation as light-induced excited state spin transition (LIESST) systems. ${ }^{23}$ This has prompted several investigations to elucidate the ultrafast dynamics and excited state cascade of 
photoexcited iron complexes. ${ }^{17}$ Recently, this has also been complemented by ultrafast X-ray spectroscopic investigations capable of probing local structure and spin dynamics involving the iron centre. ${ }^{24-26}$ Experimental progress together with increasingly advanced quantum chemical calculations and dynamics simulations have yielded a wealth of valuable insight into the ultrafast early deactivation dynamics in such complexes. ${ }^{27-31}$

In the last five years, a new ligand motif featuring strongly sigma-donating $N$ heterocyclic carbenes (NHCs) has been realized synthetically and demonstrated through a combination of time-resolved optical characterization and calculations to achieve dramatically improved excited state properties of iron complexes. ${ }^{32,33}$ This has provided a promising path for development also of iron-based photosensitizers for a variety of photoinduced electron transfer applications. Initial synthesis and characterization of octahedral $\mathrm{Fe}^{\mathrm{II}}$ complexes with up to four carbene ligands yielded excited charge-transfer state lifetimes of up to a few tens of picoseconds; ${ }^{34-36}$ significantly longer than the typical ultrafast decay of $\mathrm{Fe}-$ polypyridyl LIESST complexes on a few ps or faster timescale, though still quite short compared to e.g. many champion $\mathrm{Ru}^{\mathrm{II}}$ photosensitizers. This excited state lifetime improvement to a few tens of ps excited state lifetimes was sufficient for a recent proof-of-principle demonstration of efficient photoinduced interfacial electron injection from an anchored iron carbene photosensitizer to a semiconducting $\mathrm{TiO}_{2} \cdot{ }^{37}$

Another step towards more broadly useful iron-based photosensitizers was recently achieved through a synthetic design that involved full octahedral coordination using a hexa-carbene motif in a $\mathrm{Fe}(\mathrm{btz})_{3}$ complex with the chemical structure shown in Fig. 1. Interestingly, this complex was found to be sufficiently stable in both oxidation states II and III to allow both ground state absorption (Fig. 1b) as well as excited state dynamics characterization. $\mathrm{Fe}^{\mathrm{II}}$ and $\mathrm{Fe}^{\mathrm{III}}$ correspond to $3 \mathrm{~d}^{6}$ and $3 \mathrm{~d}^{5}$ electronic configurations or the iron centre, respectively. ${ }^{38,39}$ The $\mathrm{Fe}^{\mathrm{III}}(\mathrm{btz})_{3}$ complex was first reported in 2017 as a quite rare case of a $3 \mathrm{~d}^{5}$ complex exhibiting interesting photophysics, with a record $\sim 100$ ps chargetransfer excited state lifetime involving a ${ }^{2}$ LMCT state as illustrated in Fig. $2 .^{38}$ Spin-allowed decay to the doublet ground state $\left({ }^{2} \mathrm{GS}\right)$ also showed the first persistent room temperature photoluminescence $(\mathrm{PL})$ in the visible part of the
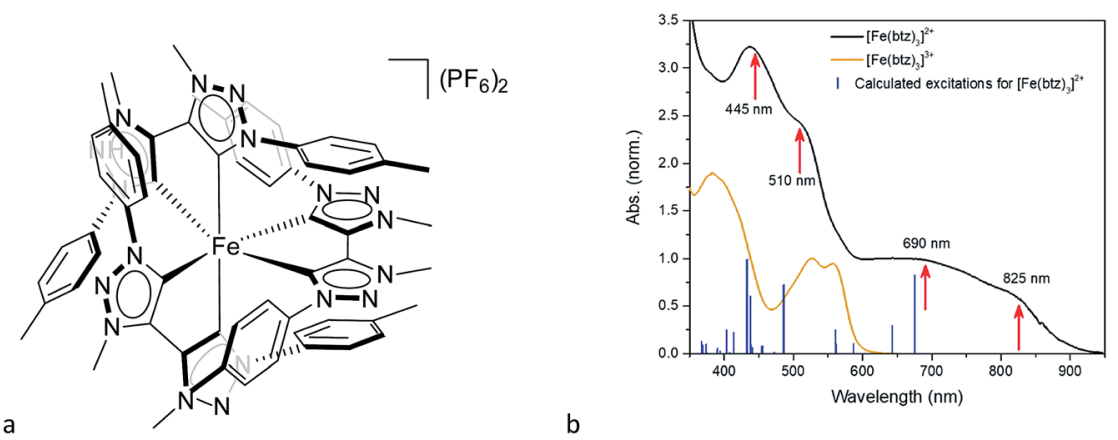

Fig. 1 (a) Chemical structure of the $\mathrm{Fe}^{\prime \prime}(\mathrm{btz})_{3}$ complex, and (b) steady state spectra of $\mathrm{Fe}^{\prime \prime}(\mathrm{btz})_{3}$ and $\mathrm{Fe}^{\prime \prime \prime}(\mathrm{btz})_{3}$ in acetonitrile. The red arrows indicate the excitation wavelengths for transient absorption experiments. 

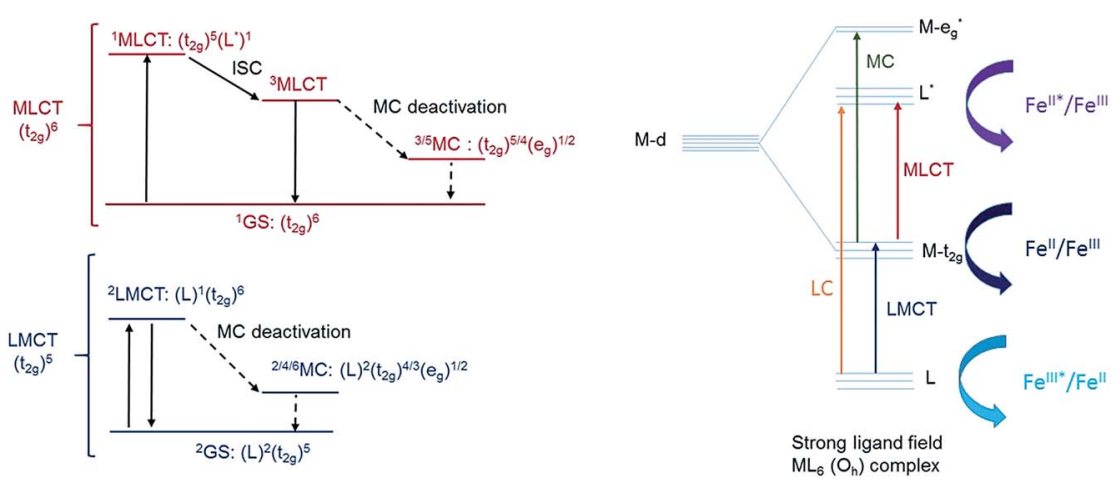

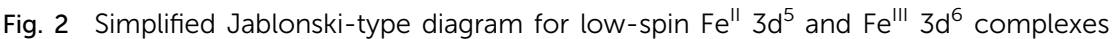
(left), together with a schematic illustration of key electronic levels and photoexcitation processes involved in the photophysics (right). Curved arrows indicate opportunities to drive subsequent bimolecular or interfacial electron transfer processes (far right).

electromagnetic spectrum from such an iron complex, albeit with a rather low PL quantum yield $\left(\Phi_{\mathrm{PL}} \sim 10^{-4}\right){ }^{38}$ The corresponding $\mathrm{Fe}^{\mathrm{II}}$ species $\mathrm{Fe}^{\mathrm{II}}(\mathrm{btz})_{3}$ was subsequently also shown to have a long room-temperature excited state lifetime of several hundred picoseconds ( $\tau \sim 500 \mathrm{ps}$ ), following a more conventional MLCT excitation scheme for a $3 \mathrm{~d}^{6}$ complex (Fig. 2). ${ }^{39}$ A new hexa-carbene $\mathrm{Fe}^{\mathrm{III}}$ complex was furthermore recently shown to feature enhanced photoluminescence from an excited CT state with a nanosecond lifetime, as well as being capable of reductive and oxidative bimolecular electron transfer reactions from the photoexcited ${ }^{2}$ LMCT state. ${ }^{40}$ The extension of the excited state lifetimes of the MLCT and LMCT charge transfer states for iron complexes into the hundreds of picosecond range and longer thus open up a range of photophysical and photochemical applications for iron carbene complexes.

It is, in this context, quite remarkable that the same strategy of increasing the $\sigma$-donation of the ligand surrounding the iron centre was effective for both $\mathrm{Fe}^{\mathrm{II}}$ and $\mathrm{Fe}^{\mathrm{III}}$ as shown in the case of $\mathrm{Fe}(\mathrm{btz})_{3}$. It is therefore interesting to develop a more comprehensive comparison of the excited state dynamics of these two types of excited state cascades. In order to achieve this, we here report new results, in particular pertaining to dynamics of $\mathrm{Fe}^{\mathrm{II}}(\mathrm{btz})_{3}$ following excitations at different excitation energies, together with some computational results detailing electronic structure properties in these photosensitizers. Together with recent time-resolved and computational results that we have reported for these complexes, this allows us to present a more comprehensive discussion focused on the ultrafast excited state dynamics of this novel type of promising photosensitizer.

\section{Methods}

\section{Experimental}

The experimental setup for recording broadband transient absorption spectra was based on a Ti:Sapphire amplified laser system (Spitfire XP Pro, Spectra Physics) operating at a $1 \mathrm{kHz}$ repetition rate, producing $\sim 80 \mathrm{fs}$ pulses centered at $796 \mathrm{~nm}$. The pump beam was tuned by an optical parametric amplifier (TOPAS C, 
Light Conversion) to excite the sample at various wavelengths (445 nm, $510 \mathrm{~nm}$, $690 \mathrm{~nm}$, and $825 \mathrm{~nm}$ ) with typical fluency $\sim 3 \times 10^{15}$ photons per pulse per $\mathrm{cm}^{2}$ and maximum fluency not exceeding $1 \times 10^{16}$ photons per pulse per $\mathrm{cm}^{2}$ which is well below absorption saturation density $\sim 5 \times 10^{17}$ photons per $\mathrm{cm}^{2}$. A power dependency check was also performed by varying the excitation intensity by a factor of 25 (100 to $2500 \mu \mathrm{W}$ ) with no deviation of the observed $\Delta A$ signal from linear behaviour. A super-continuum white-light was used as probe, generated by focusing the NIR signal from a TOPAS C into a $5 \mathrm{~mm}$ sapphire plate. The desired timing between excitation and probe pulses was achieved using a computercontrolled delay line (Aerotech, $10 \mathrm{~ns}$ ). The pump and the probe beams were overlapped on the sample with their relative polarization set to the magic-angle $\left(54.7^{\circ}\right)$ by a Berek polarization compensator placed in the pump beam path. The sample was placed in a quartz $1 \mathrm{~mm}$ path length cuvette with an automated sample mover to avoid sample photodamage, which was also checked by measuring the absorption spectra of the sample before and after each experiment. The probe and reference beams were collimated on the entrance aperture of a prism-based, double-beam spectrograph, and detected by a double diode-array detection system (Pascher Instruments). Correction of the data as well as fitting of the transient absorption datasets was carried out using both an in-house written Python routine and the analysis software DAFit (Pascher Instruments) and the individual kinetics were fitted using the Origin software.

\section{Computational}

First principles quantum chemical calculations have been performed at the Density Functional Theory (DFT) and time-dependent DFT (TD-DFT) levels of theory for ground- and excited-state properties, respectively. The presented computational results were obtained using the B3LYP* modification ${ }^{41}$ of the standard B3LYP hybrid density functional ${ }^{42}$ and employing standard 6-311G basis sets with polarization functions, ${ }^{43}$ as well as a polarizable continuum solvation model for acetonitrile. ${ }^{44}$ All calculations were performed using the Gaussian 09 program package. ${ }^{45}$

\section{Results}

In this results section, we first consider the linear absorption spectra of both $\mathrm{Fe}^{\mathrm{II}}(\mathrm{btz})_{3}$ and $\mathrm{Fe}^{\mathrm{III}}(\mathrm{btz})_{3}$ as a basis for the subsequent discussions. Next, new timeresolved optical spectroscopy results for the excited state dynamics of $\mathrm{Fe}(\mathrm{btz})_{3}$ are presented. In particular, we present new transient absorption (TA) results for the $\mathrm{Fe}^{\mathrm{II}}(\mathrm{btz})_{3}$ complex in acetonitrile following excitations at different energies. Finally, absorption spectra are complemented by a presentation of selected results from quantum chemical calculations of both $\mathrm{Fe}^{\mathrm{II}}$ and $\mathrm{Fe}^{\mathrm{II}}$ oxidation states of this complex. Together, this forms a basis for a comparative analysis of the excited state dynamics of this complex in the complementary $\mathrm{Fe}^{\mathrm{II}}\left(3 \mathrm{~d}^{6}\right)$ and $\mathrm{Fe}^{\mathrm{III}}$ $\left(3 \mathrm{~d}^{5}\right)$ oxidation states presented in the subsequent discussion below.

\section{Linear absorption}

The linear absorption spectra of $\mathrm{Fe}^{\mathrm{II}}(\mathrm{btz})_{3}$ and $\mathrm{Fe}^{\mathrm{III}}(\mathrm{btz})_{3}$, shown in Fig. 1b, clearly demonstrate that, in contrast to many other TMCs, this new class of Fe-carbene 
TMCs exhibit two distinct CT absorption bands on the red side of the strong ligand-based absorption below $\sim 350 \mathrm{~nm}$. There are, furthermore, indications of several sub-band features of vibrational or electronic nature that appear as shoulders or double peaks in the CT regions of the absorption spectra. Additionally, the blue CT band in $\mathrm{Fe}^{\mathrm{II}}(\mathrm{btz})_{3}$ has at least twice as strong maximum absorption strength compared to the red band for both oxidation states. The measured CT absorption bands agree qualitatively with the calculated singletsinglet TD-DFT spectrum for $\mathrm{Fe}^{\mathrm{II}}(\mathrm{btz})_{3}$ presented as sticks for comparison.

\section{Transient absorption}

Transient absorption data has been recorded for the $\mathrm{Fe}^{\mathrm{II}}(\mathrm{btz})_{3}$ complex as shown in Fig. 3. In particular, this includes transient data following excitations at $445 \mathrm{~nm}, 510 \mathrm{~nm}, 690 \mathrm{~nm}$, and $825 \mathrm{~nm}$, complementing the published data for excitation at $800 \mathrm{~nm}$ at key points in the Fe(II) absorption spectrum (Fig. 1). ${ }^{39}$ The excitations into different parts of the visible absorption spectrum probe the

a

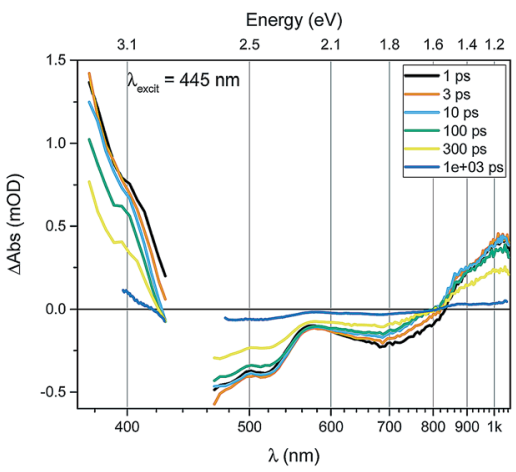

c

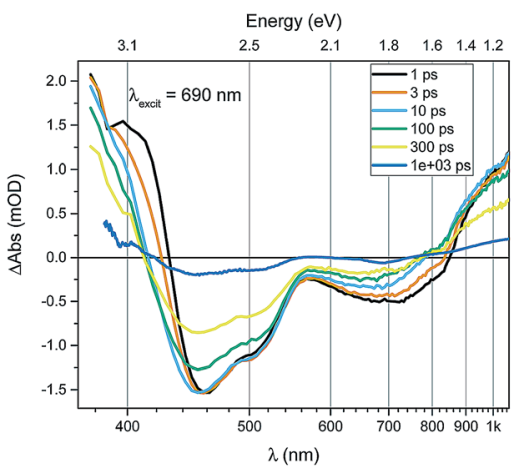

b

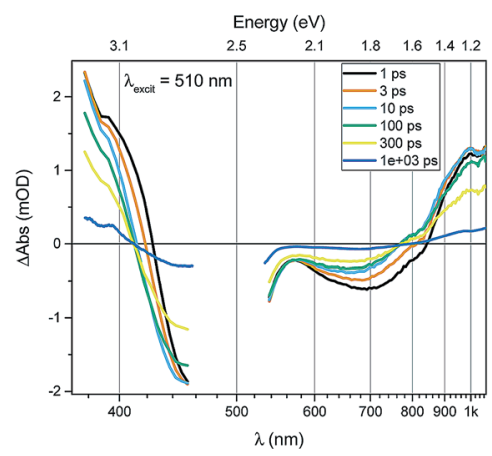

d

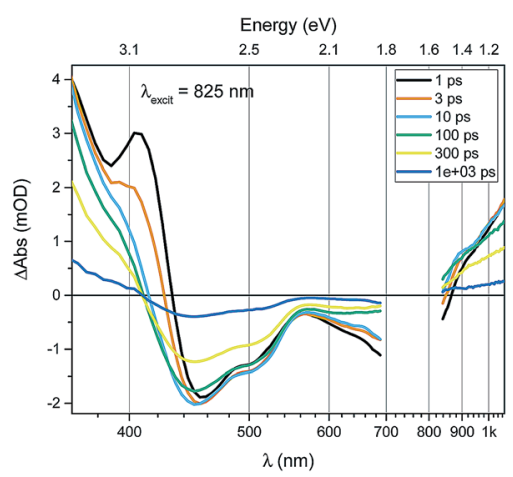

Fig. 3 Transient absorption spectra of Fe" (btz) 3 as measured, after correction for GVD (chirp) and background subtraction. Spectra were recorded following excitations at (a) $445 \mathrm{~nm}$, (b) $510 \mathrm{~nm}$, (c) $690 \mathrm{~nm}$ and (d) $825 \mathrm{~nm}$, with excitation densities of $2.4 \times 10^{15}, 3.7$ $\times 10^{15}, 3.3 \times 10^{15}$ and $1.1 \times 10^{16}$ photons per pulse per $\mathrm{cm}^{2}$, respectively. Excitation scatter region is omitted for clarity. For all panels the $x$-axis (wavelength) is in reciprocal values (linear steps in energy). 
dynamics following excitations into CT states of different electronic nature and with different initial excess energies compared to the absorption threshold.

Several main features are recognizable throughout as relatively persistent features that survive the full dynamics, also for the different excitation energies. TA dynamics are brought out further in the analysis shown in Fig. 4, which compares the spectral shape of the TA at 1 and 20 ps for all excitation energies after accounting for the excitation densities.

The main spectral features for all the four excitation energies are similar to what was recently outlined for $800 \mathrm{~nm}$ excitation in our paper introducing this complex. ${ }^{39}$ Briefly, this covers broad ground state bleach (GSB) features similar to an inverted absorption spectrum in the $c a 450-800 \mathrm{~nm}$ region of the spectrum, together with strong excited state absorption (ESA) in the blue part of the spectrum giving a positive signal above $c a 425 \mathrm{~nm}$ as well as some ESA in the red part of the spectrum that turns positive beyond the GSB region for wavelengths longer than $c a 800 \mathrm{~nm}$. The blue ESA band is apparently relatively strong, as the positive TA signal is observed in the spectral range where one would expect a pronounced bleach signal based on the linear absorption spectrum. Clearly, the overlapping ESA band must have a sharp rise towards the blue to overshoot the expected bleach shoulder, and instead give rise to a strong net positive TA signal.

A closer look at the spectral features in Fig. 3 and 4 reveals some noticeable similarities and differences in terms of the detailed shape and evolution of these TA spectra. The $510 \mathrm{~nm}$ and $690 \mathrm{~nm}$ mid-CT band spectra are, in particular, very similar throughout. The TA results for all the excitations furthermore show a qualitatively similar GSB to the TA for the recently reported energy excitation at $800 \mathrm{~nm}$. There is a noticeable reduction in the intensity of the ESA feature at $\sim 408 \mathrm{~nm}$ at early timescales for the higher energy excitations compared to the corresponding features for the lowest excitation energy at $825 \mathrm{~nm}$. There is, however, little further spectral evolution after the first $20 \mathrm{ps}$, as evident for excitations at all four excitation energies. Thus, this behaviour is very similar for the higher energy excitations compared to what has already been established for excitation at $800 \mathrm{~nm} .{ }^{39}$ It is interesting to note that although similar, the main

$\mathbf{a}$

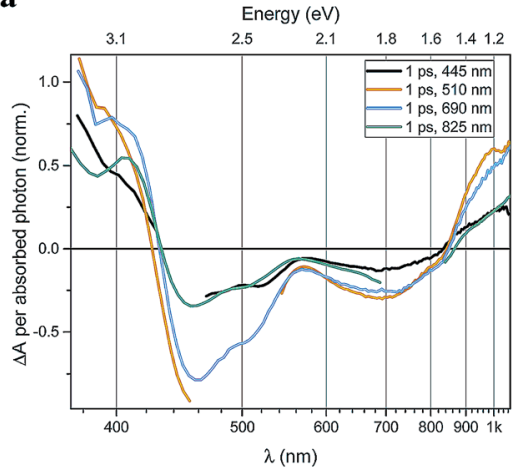

b

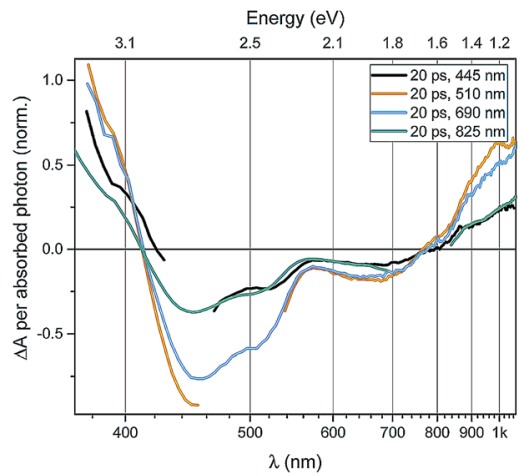

Fig. 4 Transient absorption spectra of Fe" (btz) 3 at (a) 1 ps, and (b) 20 ps normalized by the number of absorbed photons per $\mathrm{cm}^{2}$ at each respective excitation wavelength. The $x$-axis (wavelength) is in reciprocal values (linear steps in energy). 
features of the differential spectra appear to have excitation energy dependent amplitudes.

As a final step in the analysis of the excitation energy dependent dynamics, we have investigated different aspects of the multi-exponential kinetics for different combinations of pump and probe wavelengths in further detail as shown in Fig. 5, together with an associated summary of selected key kinetics components listed in Table 1. The single kinetic analysis is focused on identifying specific decay component in the TA data. As some of the decay components only contribute significantly at some probe wavelengths it is not always possible to fit all the time regions in one kinetic with current $\mathrm{S} / \mathrm{N}$ ratio in order to receive satisfactory fits. Thus only selected unambiguous components are listed in Table 1 . It can be generally noted that the dynamics in several places show signs of an ultrafast component on a sub-ps timescale that is potentially interesting in terms of ISC etc, but in all transient spectra and kinetics discussed in this study, we do not discuss further the very early dynamics that occur during the first few 100s of fs due to rather strong contribution of the coherent and solvent related experimental artefacts. For excitation energies into both main charge-transfer bands, the major part of the decay originates from a long-term component with $\tau \sim 540$ ps, as e.g. clearly evident for the $1000 \mathrm{~nm}$ kinetics for all excitation energies, and overall in good agreement with what we recently reported for excitation at $800 \mathrm{~nm}$. There is also clear evidence for a $\sim 3$ ps component at selected spectral regions in the more comprehensive TA obtained here, as seen e.g. for the $420 \mathrm{~nm}$ probes with different excitation energies presented in panels Fig. $5 \mathrm{c}$ and d; again in broad agreement with the results in our initial study based on $800 \mathrm{~nm}$ excitation. We also identify an additional new kinetic component with $\tau \sim 50$ ps as part of the early dynamics. This component is most clearly pronounced in the displayed TA data for the $408 \mathrm{~nm}$ kinetics following $825 \mathrm{~nm}$ excitation while appearing gradually less pronounced for higher energy excitations. Overall, the kinetics measured at different excitations thus look qualitatively similar, but the contribution of the different decay components is quantitatively distinct. For example, all kinetics look very similar as probed at $1000 \mathrm{~nm}$, while there are clear differences in the kinetics probed at $408 \mathrm{~nm}$ for the different excitation energies. Some implications of the rich dynamics observed here is further considered in the discussion below.

\section{Electronic structure}

Quantum chemical calculations performed at the DFT and TD-DFT level of theory provide information about the electronic states involved in the excited state cascade. Some key results were presented for both $\mathrm{Fe}^{\mathrm{III}}(\mathrm{btz})_{3}$ and $\mathrm{Fe}^{\mathrm{II}}(\mathrm{btz})_{3}$ already as part of the initial characterization of the respective species and their excited state properties. ${ }^{38,39}$ Selected computational results, including some new information, are presented here, to put results from both species on common ground to facilitate a common discussion about the similarities and differences in fundamental electronic structure properties pertinent for understanding the excited state dynamics.

First, it can be noted that the change in oxidation state from $\mathrm{Fe}^{\mathrm{II}}$ to $\mathrm{Fe}^{\mathrm{III}}$ has a profound influence both on the ground state electronic structure and the excited state manifold. The $\mathrm{Fe}^{\mathrm{II}}$ carbene complexes are characterized as a regular 
a

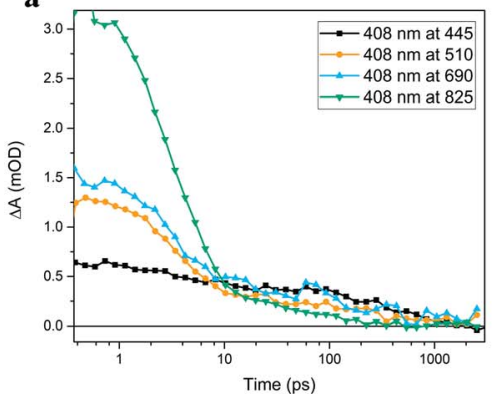

c
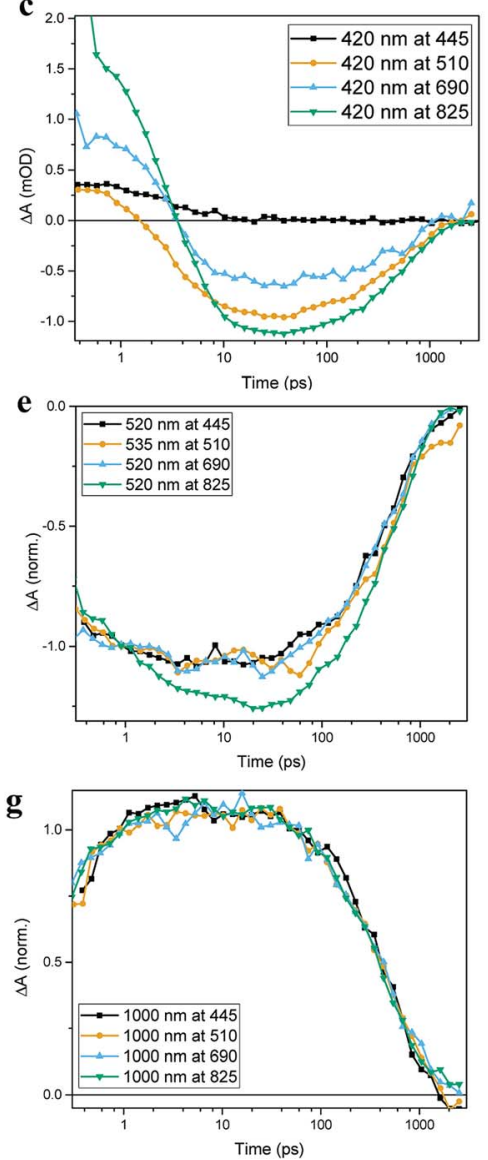

b
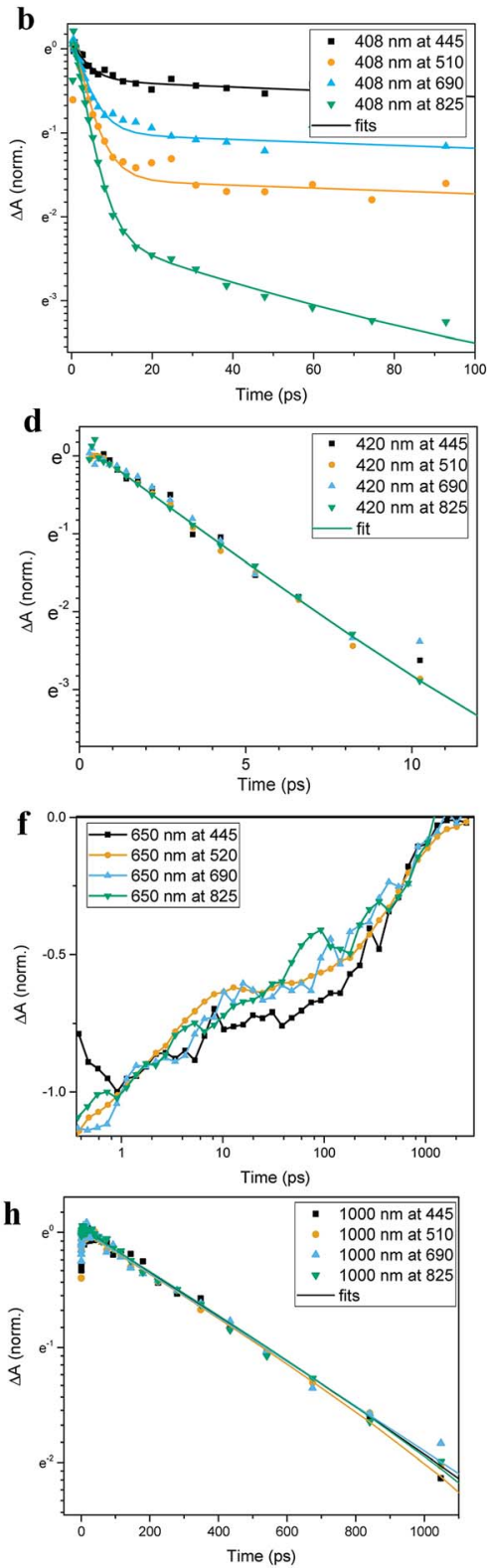

Fig. 5 Comparison of Fe" (btz) 3 kinetics following excitations at different wavelengths. Results are shown for kinetics recorded at (a and b) $408 \mathrm{~nm}$, (c and d) $420 \mathrm{~nm}$, (e) $520 \mathrm{~nm}$, (f) $650 \mathrm{~nm}$, and (g and h) $1000 \mathrm{~nm}$ following excitations at $445 \mathrm{~nm}, 510 \mathrm{~nm}, 690 \mathrm{~nm}$, and $825 \mathrm{~nm}$. Solid lines represent multi-exponential fits. Note that graphs in panels (a), (c), (e), (f) and (g) are plotted in $\Delta A$ (lin)/time (log) scale while graphs in panels (b), (d), and (h) are shown in $\Delta A(\ln ) /$ time (lin) scale. All kinetics except for panels (a) and (c) have been normalised for visual clarity.

low-spin $3 \mathrm{~d}^{6}$ species, i.e. with an electronic configuration of $\left(\mathrm{t}_{2 \mathrm{~g}}\right)^{6}$ that is similar e.g. to many well-known $\mathrm{Ru}^{\mathrm{II}}$ polypyridyl complexes. ${ }^{2}$ This features a singlet ground state and ${ }^{1 / 3}$ MLCT excited states. For traditional $\mathrm{Fe}^{\mathrm{II}}$ complexes with 
Table 1 Key kinetic components from multiexponential fits of TA data for different excitation and probe wavelengths

\begin{tabular}{|c|c|c|c|c|}
\hline$\lambda_{\text {excitation }}(\mathrm{nm})$ & $\lambda_{\text {probe }}(\mathrm{nm})$ & $\tau_{1}(\mathrm{ps})$ & $\tau_{2}(\mathrm{ps})$ & $\tau_{3}(\mathrm{ps})$ \\
\hline $445,510,690,825$ & 1000 & & & $540 \pm 35$ \\
\hline $445,510,690,825$ & 420 & $3.2 \pm 0.10$ & & \\
\hline $445,510,690$ & 408 & $3.8 \pm 0.81$ & & $540 \pm 35$ \\
\hline 825 & 408 & $3.5 \pm 0.36$ & $54.0 \pm 5.67$ & $540 \pm 35$ \\
\hline
\end{tabular}

weaker LFS, initial excitation into MLCT excited states have been extensively discussed to decay to low-energy metal centred triplet and quintet states $\left({ }^{3} \mathrm{MC}\right.$ and ${ }^{5} \mathrm{MC}$ ) on ultrafast timescales. ${ }^{17,21,22,27,46}$ This is typically followed by slower ground state recovery from meta-stable MC states on the timescale of hundreds of picosecond or slower. ${ }^{47}$ Dramatically different excited state dynamics has instead been observed for $\mathrm{Fe}^{\mathrm{II}}$ carbene complexes, including assignment of extended excited state lifetimes of ${ }^{3}$ MLCT states of $\sim 10$ ps or longer, and followed by fast ground state recovery on similar timescales. The changes in excited state dynamics are largely attributed to a strong destabilization of the metal centred states in the iron carbene complexes due to the strong sigma-donating nature of the carbenes. ${ }^{\mathbf{1 4}}$ This altered photophysics also allowed a recent demonstration of efficient electron injection into a $\mathrm{TiO}_{2}$ substrate. ${ }^{37}$ The electronic structure for $\mathrm{Fe}^{\mathrm{II}}(\mathrm{btz})_{3}$ features a characteristic ${ }^{3}$ MLCT-like spin density for the photofunctional chargetransfer state (Fig. 6a).

The electronic structure properties of $\mathrm{Fe}^{\mathrm{III}}(\mathrm{btz})_{3}$ can be understood in simple terms as originating from a one-electron oxidation of the $\mathrm{Fe}^{\mathrm{II}}(\mathrm{btz})_{3}$ complex in which one electron is removed from the iron center so that it takes on what could recently been characterized as a low-spin $3 \mathrm{~d}^{5}$ electronic configuration, i.e. with a $\left(t_{2 g}\right)^{5}$ population on iron in quasi-octahedral symmetry notation (Fig. 2). From this follows that the ground state is a doublet (Fig. 6b), and that the excited state manifold will consist of doublet, quartet, and hextet excited states (limiting the number of unpaired electrons to five for simplicity). With a hole in the $\left(t_{2 g}\right)$ levels, the lowest $\mathrm{Fe}^{\mathrm{III}}(\mathrm{btz})_{3}$ excitation was characterized as a doublet ligand-to-metal charge transfer state. ${ }^{38}$ Metal centered quartet and hextet states could also be identified computationally, and involve population of $\mathrm{e}_{\mathrm{g}}$-levels on the iron center. Such intermediate- and high-spin MC states are naturally stabilized by elongated metal-ligand bonds, similar to the MC states for $\mathrm{d}^{6}$ complexes. ${ }^{1}$ Calculated spin density plots of such ${ }^{4} \mathrm{MC}$ and ${ }^{6} \mathrm{MC}$ states for $\mathrm{Fe}^{\mathrm{III}}(\mathrm{btz})_{3}$ are shown in Fig. $6 \mathrm{c}$ and d panels, respectively.

It is thus clear that the $\mathrm{Fe}^{\mathrm{II}}$ and $\mathrm{Fe}^{\mathrm{III}}$ forms of iron carbene complexes like $\mathrm{Fe}(\mathrm{btz})_{3}$ differ fundamentally in their basic electronic properties, excitations, and excited state manifolds. In particular, the altered electronic structure effectively yields a reversal of the fundamental direction of the lowest charge-transfer excitations, and while there are metal centered scavenger states that facilitate excited state deactivation for both oxidation states, there are fundamental differences in the spin properties of the states and state crossings involved in the excited state cascade. It is therefore interesting to compare the excited state dynamics for the same complex in the two different oxidation states. 
a

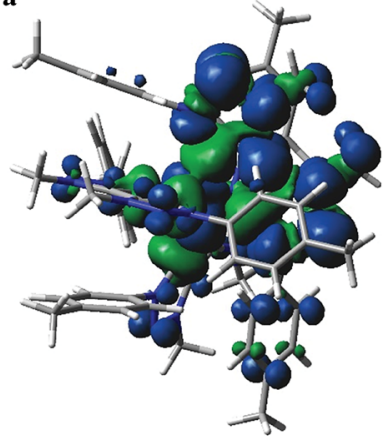

c

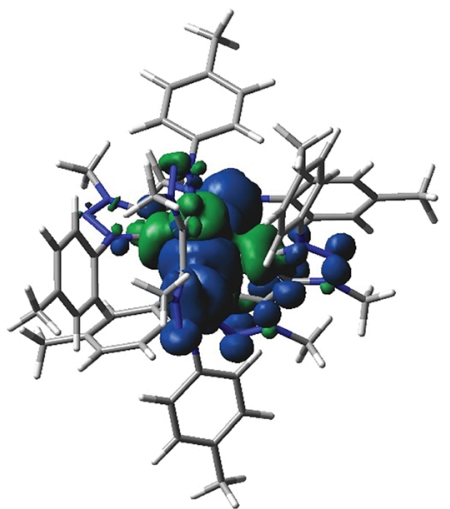

b

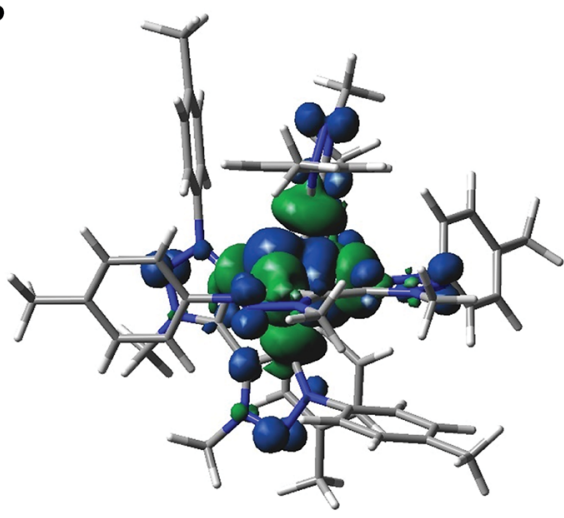

d

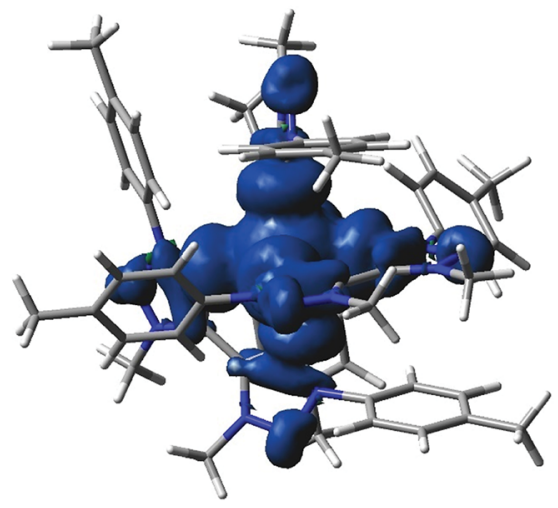

Fig. 6 Spin density plots (isovalue 0.0004 ) of selected $\mathrm{Fe}^{\prime \prime}(\mathrm{btz})_{3}$ and $\mathrm{Fe}^{\prime \prime \prime}(\mathrm{btz})_{3}$ states from B3LYP*/6-311G(d)|MeCN calculations. Specific oxidation and electronic state assign-

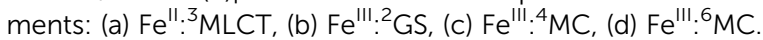

One potentially promising aspect of the photophysics of the $\mathrm{Fe}^{\mathrm{III}}(\mathrm{btz})_{3}$ complex for photosensitizer applications is that the excited state lifetime of the ${ }^{2}$ LMCT state was characterized experimentally to be largely unaffected by the excitation energy over a significant part of the visible absorption band (probed between ca 400 and $550 \mathrm{~nm}) .{ }^{39}$ This facilitates for the complex to perform well as a photosensitizer for a broad range of solar illumination. Here, the dynamics of the $\mathrm{Fe}^{\mathrm{II}}(\mathrm{btz})_{3}$ complex is also further explored experimentally via TA to enable a comparative discussion of the photophysics of the two oxidation states. It is in this context relevant to consider the excited state manifold that can be accessed at different excitation energies also for this $\mathrm{Fe}^{\mathrm{II}}$ complex. Fig. 7 provides a graphical illustration of singlet and triplet excited states in the accessible energy region obtained from singletsinglet and singlet-triplet TD-DFT calculations, respectively.

\section{Discussion}

Conventionally, the main electronic states considered for excited state evolution in $\mathrm{d}^{6} \mathrm{TMCs}$ include the singlet ground state $\left({ }^{1} \mathrm{GS}\right),{ }^{1} \mathrm{MLCT},{ }^{3} \mathrm{MLCT},{ }^{3} \mathrm{MC}$ and, for 


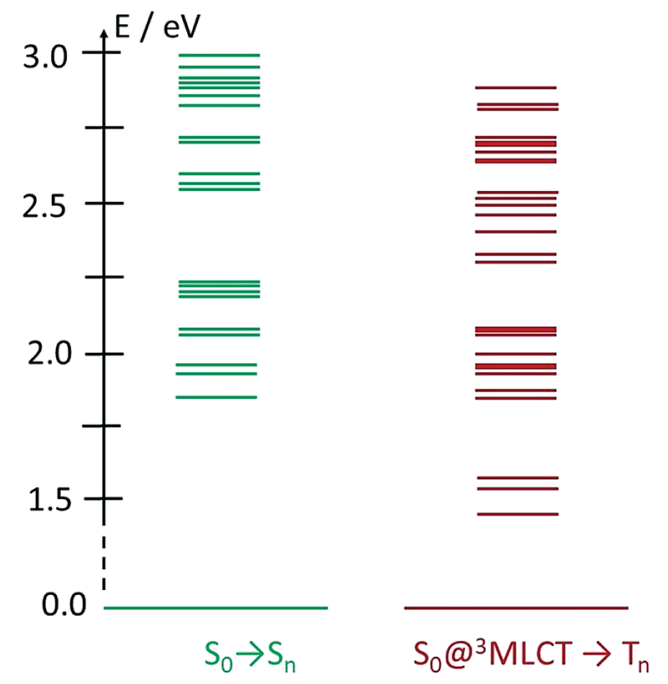

Fig. 7 TD-DFT excited state calculations for Fe" $(b t z)_{3}$. Shown excitations include singlet excitations at SO geometry (left, green), and triplet excitations from the SO state at the ${ }^{3}$ MLCT geometry (right, red). Energies and assignments from Chábera et al. ${ }^{39}$

complexes with weak LFS, ${ }^{5} \mathrm{MC}$, and in many cases modelling excited state dynamics as an interplay of these states has provided a satisfactory picture of the overall spectral changes associated with the excited state evolution including depopulation of the initial charge transfer state (loss of ESA) and ground state recovery (loss of GSB).

Our results for $\mathrm{Fe}(\mathrm{btz})_{3}$ provide evidence that a more sophisticated description may generally be needed for a comprehensive understanding of the photoinduced dynamics of iron carbenes. A strong indication of the rather special features of $\mathrm{Fe}(\mathrm{btz})_{3}$ is apparent already from analysis of the absorption spectra. In contrast to MLCT absorption of many typical TMCs, we record two well-resolved CT transitions centered at 450 and $700 \mathrm{~nm}$, assigned to population of two different CT bands in $\mathrm{Fe}^{\mathrm{II}}(\mathrm{btz})_{3}$. For $\mathrm{Fe}^{\mathrm{II}}$, one can furthermore identify two high-energy shoulders at $\sim 520$ and $\sim 825 \mathrm{~nm}$ for the two ${ }^{1}$ MLCT absorption bands, respectively. We can attribute this rich CT absorption appearance as an effect of the strong $\sigma$-donating effect of the carbene ligands which causes an up-shift in the energy of the highest occupied metal-based $t_{2 g}$ levels of the ground state (correlated also to a shift towards more negative ground state reduction potentials). ${ }^{1}$ This shifts the CT transition energies towards red to the extent that more of the CT manifold appears clearly below the more intense intra-ligand transitions. This observation is in very good agreement with the predictions of our TD-DFT calculations which, as outlined above, indicate that there is a broad range of charge-transfer excitations that cover the visible spectral region in the experimental linear absorption spectrum.

Having clearly distinct CT bands that are well separated in energy and furthermore have very different transition strength, provides an opportunity to investigate to what extent the dynamics following excitation to different CT states are similar or different. In the following discussion, we will first consider 
separately $\mathrm{Fe}^{\mathrm{II}}$ and $\mathrm{Fe}^{\mathrm{III}}$ cases before our final conclusions relating to the emergence of a more comprehensive view of the photoinduced dynamics of the iron carbenes.

As described in the results section, we observe differences in the excited state dynamics in $\mathrm{Fe}^{\mathrm{II}}(\mathrm{btz})_{3}$ after excitation into the two ${ }^{1}$ MLCT bands. In particular, we observe some clear changes of the TA spectral shapes for different excitations, as exemplified by the more pronounced $\sim 408$ band in the ESA from excitations at low excitation energy at early timescales. Following the established picture of early $\mathrm{Fe}^{\mathrm{II}}$ excited state evolution, we assume very efficient depopulation of the ${ }^{1}$ MLCT due to fast ISC on a sub-picosecond timescale (not resolved in this study). ${ }^{46}$ Our observations suggest that excitation into different parts of the ${ }^{1}$ MLCT bands leads to different population dynamics and branching ratio between the ${ }^{3} \mathrm{MLCT}$ and ${ }^{3} \mathrm{MC}$ excited states. Initially hot conversion of ${ }^{3} \mathrm{MLCT}$ into ${ }^{3} \mathrm{MC}$ probably does not occur for delay times longer than $\sim 1 \mathrm{ps}$. In agreement with our previous assignments for excitation at $\sim 800 \mathrm{~nm}$, we tentatively associate some changes to the blue part of the ESA at early times (see Fig. 3-5) mainly with intramolecular vibration relaxation (IVR). ${ }^{39}$ Hot population of the energetically accessible ${ }^{3} \mathrm{MC}$ is likely to be limited to the early times prior to such IVR. It is tempting to conclude that population of the ${ }^{3} \mathrm{MC}$ occurs from ${ }^{1} \mathrm{MLCT}$ or "hot" ${ }^{3}$ MLCT whereas the relaxed ${ }^{3}$ MLCT has a lower probability of converting into ${ }^{3} \mathrm{MC}$. This interpretation of the very fast excited state processes is visualized schematically in Fig. 8. This suggests that the efficiency of the ${ }^{3} \mathrm{MC}$ population is very sensitive to the exact crossings of the ${ }^{1}$ MLCT and ${ }^{3}$ MLCT with the ${ }^{3} \mathrm{MC}$ state in

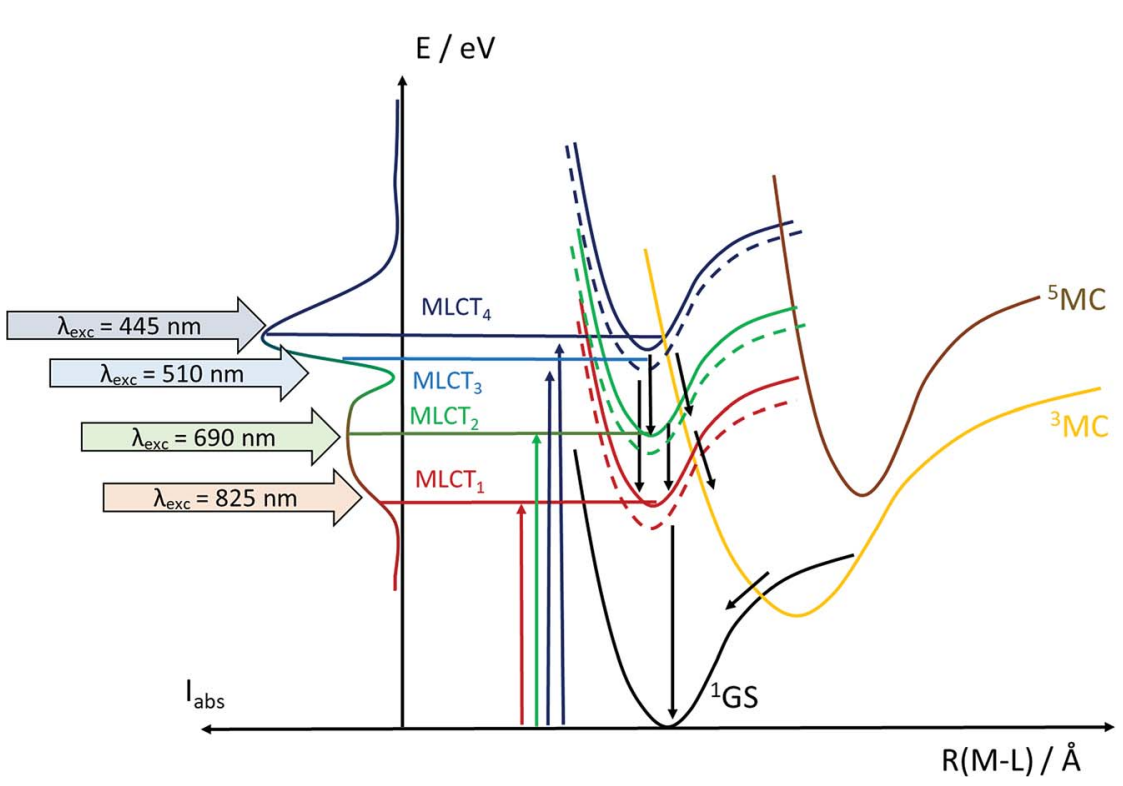

Fig. 8 Excited state manifold in the charge-transfer excitation energy region for Fe" $(\mathrm{btz})_{3}$, indicating the different excitation energies/wavelengths employed experimentally to probe the excited state dynamics (red, green, light blue and blue arrows), as well as selected potential decay paths (black arrows). Singlet and triplet forms of representative MLCT states in the charge-transfer manifold energy region are distinguished as solid and dashed lines, respectively. 
terms of the activation energy and structural rearrangement associated with such a transition.

Finally, irrespective of the rate and amplitude of this few tens of ps component, the overall relaxation of the excited complexes to the ground state occurs with the same rate within our signal-to-noise $(\mathrm{S} / \mathrm{N})$. These observations agree with dynamics on a manifold of ${ }^{3}$ MLCT excited states, including energy relaxation within this manifold that occurs on timescales up to a few tens of ps. Based on the combined information from ground state absorption, first principles calculations, and time-resolved excited state dynamics, we infer that a consistent and complete interpretation of the results require a manifold of ${ }^{1} \mathrm{MLCT}$ and ${ }^{3} \mathrm{MLCT}$ excited states to be considered.

Taken together, the simultaneous excitation and probing of different parts of the CT manifold presented in this investigation provides a first glimpse of rich, and in some aspects band-selective, dynamics in these iron carbene complexes with remarkably long-lived ( $>100 \mathrm{ps}$ ) excited states. From the current understanding of the excited state manifold of these unconventional iron complexes the full dynamics potentially involves a combination of several excited state processes including ultrafast electronic processes such as IVR, IC, ISC within the manifold of excited states, together with molecular and solvent structural dynamics. The current evidence from the TA dynamics suggests that it will be important to carry out further work to fully elucidate key aspects of the ultrafast mechanisms responsible for the dynamics observations, including unambiguous assignments of the ultrafast dynamics and detailed interplay between initial singlet-triplet ISC as well as the deactivation cascade from CT to MC states for a full comparison with the ultrafast LIESST dynamics in conventional Fe complexes typically characterized by ultrafast ISC and CT $\rightarrow$ MC conversion. A range of further experimental and computational investigative steps appear promising to facilitate a better understanding, including complementary optical characterization e.g. including low-temperature characterization, time-resolved optical measurements with better time-resolution in the ultrafast domain, and studies of dynamics in different solvent environments, as well as complimentary experimental techniques in different energy regimes including e.g. time-resolved X-ray dynamics, as well as more sophisticated theoretical treatments such as high-level quantum dynamics simulations.

It is finally interesting to also compare these new results for $\mathrm{Fe}^{\mathrm{II}}$ to those recently reported for the $\mathrm{Fe}^{\mathrm{III}}$ form of the $\mathrm{Fe}(\mathrm{btz})_{3}$ complex. Similar to $\mathrm{Fe}^{\mathrm{II}}$, two clearly separated transitions to CT states in the ground state absorption spectrum can be identified: a high energy band centered at $\sim 400$ and a low energy band with two peaks at 525 and $560 \mathrm{~nm}$. No significant excitation wavelength dependency in the TA spectra and kinetics was, however, noticed. It is important to note here that the excited state energy evolution does not exhibit fast and efficient ISC from the ${ }^{2}$ LMCT state. On the contrary, after optical excitation from the doublet ground state $\left({ }^{2} \mathrm{GS}\right)$ to the ${ }^{2} \mathrm{LMCT}$ state, the primary energy relaxation occurs within the same excited state. This is evident as the excited state is emissive and exhibits a rather small Stokes shift. After reaching the bottom of the ${ }^{2}$ LMCT manifold, the excited state population undergoes either radiative or non-radiative transition to the ground state in competition with activated ISC transition to ${ }^{4} \mathrm{MC}$ scavenger states. The relative efficiencies of the above listed transitions determine the 
excited state lifetime (identical to the ground state recovery time), as well as the yield of the photoluminescence.

\section{Conclusions}

Efforts to develop iron carbene photosensitizer complexes have recently yielded very significant improvements in excited state lifetimes for both conventional $3 \mathrm{~d}^{6}$ $\mathrm{Fe}^{\mathrm{II}}$ carbene complexes, and more unconventional low-spin $3 \mathrm{~d}^{5} \mathrm{Fe}^{\mathrm{III}}$ complexes. These results were obtained in rapid succession for structurally similar $\mathrm{Fe}^{\mathrm{II}}$ and $\mathrm{Fe}^{\mathrm{III}}$ complexes and appear to rely on the same ligand design strategy to maximize the strong $\sigma$-donation by hexa-carbene environments around the metal in the two different formal oxidation states of the iron center. Our results on the excited state properties and dynamics of the $\mathrm{Fe}(\mathrm{btz})_{3}$ molecule with iron in both oxidation state II and III provide an excellent opportunity to compare promising photophysics and photochemistry of iron carbene photosensitizers in the two different electronic configurations $3 \mathrm{~d}^{5}$ and $3 \mathrm{~d}^{6}$. Until recently, only a relatively limited number of $\mathrm{d}^{5}{ }^{2} \mathrm{LMCT}$ complexes, e.g. $\operatorname{Re}(\mathrm{II})$ and Tc(II), had received significant attention as potentially interesting for driving photocatalytic processes. ${ }^{4-50}$ Our combined results for the $\mathrm{Fe}(\mathrm{btz})_{3}$ complex suggest that the photophysics and photochemistry of these complexes provide interesting information regarding ultrafast dynamics following excitations into a manifold of charge-transfer excited states that may be relevant more broadly.

One key finding for the time-resolved TA dynamics of $\mathrm{Fe}^{\mathrm{II}}(\mathrm{btz})_{3}$ following higher energy excitations (from $825 \mathrm{~nm}$ to $445 \mathrm{~nm}$ excitation wavelengths) is that there remains a long-lived ground state recovery with a time constant of $c a 500$ ps independent of excitation wavelength accounting for a major part of the decay dynamics. This photophysical consistency in terms of a stable long-term excited state feature following excitation into the charge-transfer manifold of excited states/ bands is similar to what was recently shown in our investigation of the $\mathrm{Fe}^{\mathrm{III}}(\mathrm{btz})_{3}$ form (in the range of 550-400 $\mathrm{nm}$ excitation wavelengths) where there was a $c a 100$ ps long component dominating the deactivation and ground state recovery regardless of excitation energy. This common feature of the two oxidation states is potentially quite important for the photofunctionality of the iron carbenes e.g. for light-harvesting in solar energy conversion applications profiting from exposure to the full solar spectrum, and not just limited to absorption in a small spectral region.

The detailed investigation of the ultrafast dynamics of the $\mathrm{Fe}^{\mathrm{II}}(\mathrm{btz})_{3}$ complex through multi-exponential fitting of selected TA kinetics, however, also reveals some interesting additional, smaller but clear, contributions to the dynamics on timescales of a few tens of ps that was not previously discussed. The quantum chemical calculations provide general support for the presence of a manifold of low excited-state energy metal-to-ligand charge transfer states, e.g. involving electronic rearrangements either among the $\left(\mathrm{t}_{2 \mathrm{~g}}\right)^{5}$ electrons or low-energy reduced $\left(\pi_{\mathrm{btz}}^{*}\right)^{1}$ ligand orbitals in the MLCT configuration, at energies that become accessible for the higher energy excitations. It is therefore in itself not surprising that the complex excited state landscape leads to some additional fast dynamics, e.g. involving internal conversion or intersystem crossing. With the present TA and computational results, a detailed interpretation of the short-lived intermediate dynamics was not attempted. Instead, we note that given the current interest in hot dynamics in transition metal complexes in general, coupled with the 
current high interest in developing Earth-abundant photosensitizers based on iron, our new findings point to the importance of further investigations to characterize the ultrafast dynamics in this type of iron complexes with rich dynamics and extended excited state lifetimes in greater detail.

A comparison of the ultrafast charge-transfer dynamics and excited state cascade of $\mathrm{Fe}^{\mathrm{II}}$ and $\mathrm{Fe}^{\mathrm{III}}$ species finally provides a rare opportunity to compare largely complementary excited state dynamics including issues, e.g. about how internal deexcitation differ in charge-transfer excited manifolds that differ in the fundamental charge-transfer direction (MLCT versus LMCT in $\mathrm{Fe}^{\mathrm{II}}$ and $\mathrm{Fe}^{\mathrm{III}}$ species, respectively). The comparative analysis also points to fundamental differences in energy relaxation paths and state-crossings for species with fundamentally different sets of spin states (even versus odd electronic state multiplicities) that has significant impact on light-harvesting and light-emitting photofunctionality. Further studies of ultrafast dynamics of iron complexes with long-lived charge transfer excited states thus look important for providing further in-depth understanding relevant to the continued development of photofunctional iron complexes. More generally, it remains interesting to conduct investigations of the ultrafast photoinduced dynamics also for other transition metal complexes with unconventional excited state properties and novel ultrafast dynamics.

\section{Conflicts of interest}

The authors declare no conflicts of interest.

\section{Acknowledgements}

The Swedish Research Council (VR), the Swedish Energy Agency (Energimyndigheten), the Swedish Foundation for Strategic Research (SSF), and the Knut and Alice Wallenberg (KAW) Foundation are all acknowledged for financial support. The Swedish supercomputing centers LUNARC and NSC are gratefully acknowledged for providing computing facilities through the SNIC allocation system.

\section{References}

1 C. S. Ponseca, P. Chábera, J. Uhlig, P. Persson and V. Sundström, Ultrafast Electron Dynamics in Solar Energy Conversion, Chem. Rev., 2017, 117(16), 10940-11024.

2 V. Balzani, G. Bergamini, S. Campagna and F. Puntoriero, Photochemistry and Photophysics of Coordination Compounds: Overview and General Concepts, Top. Curr. Chem., 2007, 280, 1-36.

3 N. S. Lewis and D. G. Nocera, Powering the Planet: Chemical Challenges in Solar Energy Utilization, Proc. Natl. Acad. Sci. U. S. A., 2006, 103(43), 1572915735.

4 G. J. Meyer, Molecular Approaches to Solar Energy Conversion with Coordination Compounds Anchored to Semiconductor Surfaces, Inorg. Chem., 2005, 44(20), 6852-6864.

5 A. Hagfeldt and M. Graetzel, Light-Induced Redox Reactions in Nanocrystalline Systems, Chem. Rev., 1995, 95(1), 49-68. 
6 V. W.-W. Yam and K. M.-C. Wong, Luminescent Metal Complexes of D6, D8 and D10 Transition Metal Centres, Chem. Commun., 2011, 47(42), 11579.

7 L. A. Büldt and O. S. Wenger, Luminescent Complexes Made from Chelating Isocyanide Ligands and Earth-Abundant Metals, Dalton Trans., 2017, 46, 15175-15177.

8 B. Bozic-Weber, E. C. Constable and C. E. Housecroft, Light Harvesting with Earth Abundant D-Block Metals: Development of Sensitizers in DyeSensitized Solar Cells (DSCs), Coord. Chem. Rev., 2013, 257(21-22), 3089-3106.

9 O. S. Wenger, Photoactive Complexes with Earth-Abundant Metals, J. Am. Chem. Soc., 2018, 140(42), 13522-13533.

10 P. Sutra and A. Igau, Emerging Earth-Abundant (Fe, Co, Ni, Cu) Molecular Complexes for Solar Fuel Catalysis, Curr. Opin. Green Sustain. Chem., 2018, 10, 60-67.

11 B. M. Hunter, H. B. Gray and A. M. Müller, Earth-Abundant Heterogeneous Water Oxidation Catalysts, Chem. Rev., 2016, 116(22), 14120-14136.

12 T. Bessho, E. C. Constable, M. Graetzel, R. A. Hernandez, C. E. Housecroft, W. Kylberg, M. K. Nazeeruddin, M. Neuburger and S. Schaffner, An Element of Surprise-Efficient Copper-Functionalized Dye-Sensitized Solar Cells, Chem. Commun., 2008, 32, 3717-3719.

13 S. Otto, M. Grabolle, C. Förster, C. Kreitner, U. Resch-Genger and K. Heinze, $\left[\mathrm{Cr}(\mathrm{Ddpd})_{2}\right]^{3+}$ : A Molecular, Water-Soluble, Highly NIR-Emissive Ruby Analogue, Angew. Chem., Int. Ed., 2015, 54(39), 11572-11576.

14 Y. Liu, P. Persson, V. Sundström and K. Wärnmark, Fe N-Heterocyclic Carbene Complexes as Promising Photosensitizers, Acc. Chem. Res., 2016, 49, 14771485.

15 M. Abrahamsson, M. J. Lundqvist, H. Wolpher, O. Johansson, L. Eriksson, J. Bergquist, T. Rasmussen, H. C. Becker, L. Hammarström, P. O. Norrby, et al., Steric Influence on the Excited-State Lifetimes of Ruthenium Complexes with Bipyridyl-Alkanylene-Pyridyl Ligands, Inorg. Chem., 2008, 47(9), 3540-3548.

16 M. Abrahamsson, Solar Energy Conversion Using Iron Polypyridyl Type Photosensitizers - a Viable Route for the Future?, in Photochemistry, ed. A. Albini and E. Fasani, RSC, Cambridge, 2017, vol. 44, pp. 285-295, ISBN: 9781-78262-543-8, DOI: 10.1039/9781782626954.

17 J. E. Monat and J. K. McCusker, Femtosecond Excited-State Dynamics of an Iron(II) Polypyridyl Solar Cell Sensitizer Model, J. Am. Chem. Soc., 2000, 122(17), 4092-4097.

18 S. M. Fatur, S. G. Shepard, R. F. Higgins, M. P. Shores and N. H. Damrauer , A Synthetically Tunable System To Control MLCT Excited-State Lifetimes and Spin States in Iron(II) Polypyridines, J. Am. Chem. Soc., 2017, 139, 4493-4505.

19 W. Zhang, R. Alonso-Mori, U. Bergmann, C. Bressler, M. Chollet, A. Galler, W. Gawelda, R. G. Hadt, R. W. Hartsock, T. Kroll, et al., Tracking ExcitedState Charge and Spin Dynamics in Iron Coordination Complexes, Nature, 2014, 509(7500), 345-348.

20 A. Cannizzo, C. J. Milne, C. Consani, W. Gawelda, C. Bressler, F. Van Mourik and M. Chergui, Light-Induced Spin Crossover in Fe(II)-Based Complexes: The Full Photocycle Unraveled by Ultrafast Optical and X-Ray Spectroscopies, Coord. Chem. Rev., 2009, 254, 2677-2686. 
21 W. Zhang, R. Alonso-Mori, U. Bergmann, C. Bressler, M. Chollet, A. Galler, W. Gawelda, R. G. Hadt, R. W. Hartsock, T. Kroll, et al., Tracking ExcitedState Charge and Spin Dynamics in Iron Coordination Complexes, Nature, 2014, 509(7500), 345-348.

22 J. K. Mccusker and A. Vlcek, Ultrafast Excited-State Processes in Inorganic Systems, Acc. Chem. Res., 2015, 48, 1207-1208.

23 Q. Sun, S. Mosquera-Vasquez, L. M. Daku, L. Guénée, H. A. Goodwin, E. Vauthey and A. Hauser, Experimental Evidence of Ultrafast Quenching of the 3 MLCT Luminescence in Ruthenium(II) Tris-Bipyridyl Complexes via a 3dd State, J. Am. Chem. Soc., 2013, 135, 13660-13663.

24 M. Chergui and E. Collet, Photoinduced Structural Dynamics of Molecular Systems Mapped by Time-Resolved X-ray Methods, Chem. Rev., 2017, 117(16), 11025-11065.

25 T. J. Penfold, M. Pápai, T. Rozgonyi, K. B. Møller and G. Vankó, Probing Spinvibronic Dynamics Using Femtosecond X-Ray Spectroscopy, Faraday Discuss., 2016, 194(0), 731-746.

26 D. Leshchev, T. C. B. Harlang, L. A. Fredin, D. Khakhulin, Y. Liu, E. Biasin, M. G. Laursen, G. E. Newby, K. Haldrup, M. M. Nielsen, et al., Tracking the Picosecond Deactivation Dynamics of a Photoexcited Iron Carbene Complex by Time-Resolved X-Ray Scattering, Chem. Sci., 2018, 9, 405-414.

27 W. Zhang and K. J. Gaffney, Mechanistic Studies of Photoinduced Spin Crossover and Electron Transfer in Inorganic Complexes, Acc. Chem. Res., 2015, 48, 1140-1148.

28 T. J. Penfold, E. Gindensperger, C. Daniel and C. M. Marian, Spin-Vibronic Mechanism for Intersystem Crossing, Chem. Rev., 2018, 118, 6975-7025.

29 C. Sousa, M. Alías, A. Domingo and C. de Graaf, Deactivation of Excited States in Transition-Metal Complexes: Insight from Computational Chemistry, Chem.-Eur. J., 2019, 25(5), 1152-1164.

30 J. Nance, D. N. Bowman, S. Mukherjee, C. T. Kelley and E. Jakubikova, Insights into the Spin-State Transitions in $\left[\mathrm{Fe}(\mathrm{Tpy})_{2}\right]^{2+}$ : Importance of the Terpyridine Rocking Motion, Inorg. Chem., 2015, 54, 11259-11268.

31 I. M. Dixon, F. Alary, M. Boggio-Pasqua and J.-L. Heully, The $\left(\mathrm{N}_{4} \mathrm{C}_{2}\right)^{2-}$ Donor Set as Promising Motif for Bis(Tridentate) Iron(II) Photoactive Compounds, Inorg. Chem., 2013, 52(23), 13369-13374.

32 Y. Liu, P. Persson, V. Sundström and K. Wärnmark, Fe N-Heterocyclic Carbene Complexes as Promising Photosensitizers, Acc. Chem. Res., 2016, 49(8), 14771485.

33 L. Liu, T. Duchanois, T. Etienne, A. Monari, M. Beley, X. Assfeld, S. Haacke and P. C. Gros, A New Record Excited State 3 MLCT Lifetime for Metalorganic Iron(II) Complexes, Phys. Chem. Chem. Phys., 2016, 18(18), 12550-12556.

34 Y. Liu, T. Harlang, S. E. Canton, P. Chábera, K. Suárez-Alcántara, A. Fleckhaus, D. A. Vithanage, E. Göransson, A. Corani, R. Lomoth, et al., Towards LongerLived Metal-to-Ligand Charge Transfer States of Iron(II) Complexes: An NHeterocyclic Carbene Approach, Chem. Commun., 2013, 49(49), 6412-6414.

35 Y. Liu, K. S. Kjaer, L. A. Fredin, P. Chabera, T. Harlang, S. E. Canton, S. Lidin, J. Zhang, R. Lomoth, K. E. Bergquist, et al., A Heteroleptic Ferrous Complex with Mesoionic Bis(1,2,3-Triazol-5-Ylidene) Ligands: Taming the MLCT Excited State of Iron(II), Chem.-Eur. J., 2015, 21(9), 3628-3639. 
36 L. A. Fredin, M. Papai, E. Rozsalyi, G. Vanko, K. Warnmark, V. Sundstrom and P. Persson, Exceptional Excited-State Lifetime of an Iron(II)-N-Heterocyclic Carbene Complex Explained, J. Phys. Chem. Lett., 2014, 5(12), 2066-2071.

37 T. C. B. Harlang, Y. Liu, O. Gordivska, L. A. Fredin, C. S. Ponseca, P. Huang, P. Chábera, K. S. Kjaer, H. Mateos, J. Uhlig, et al., Iron Sensitizer Converts Light to Electrons with 92\% Yield, Nat. Chem., 2015, 7(11), 883-889.

38 P. Chabera, Y. Liu, O. Prakash, E. Thyraug, A. El Nahhas, A. Honarfar, S. Essen, L. A. Fredin, T. C. B. Harlang, K. S. Kjaer, et al., A Low-Spin Fe(III) Complex with 100-Ps Ligand-to-Metal Charge Transfer Photoluminescence, Nature, 2017, 543, 695.

39 P. Chábera, K. S. Kjaer, O. Prakash, A. Honarfar, Y. Liu, L. A. Fredin, T. C. B. Harlang, S. Lidin, J. Uhlig, V. Sundström, et al., $\mathrm{Fe}^{\mathrm{II}} \mathrm{Hexa} \mathrm{N}-$ Heterocyclic Carbene Complex with a 528 Ps Metal-To-Ligand ChargeTransfer Excited-State Lifetime, J. Phys. Chem. Lett., 2018, 9(3), 459-463.

40 K. S. Kjær, N. Kaul, O. Prakash, P. Chábera, N. W. Rosemann, A. Honarfar, O. Gordivska, L. A. Fredin, K.-E. Bergquist, L. Häggström, et al., Luminescence and Reactivity of a Charge-Transfer Excited Iron Complex with Nanosecond Lifetime, Science, 2019, 363(6424), 249-253.

41 M. Reiher, O. Salomon and B. A. Hess, Reparameterization of Hybrid Functionals Based on Energy Differences of States of Different Multiplicity, Theor. Chem. Acc., 2001, 107, 48-55.

42 A. D. Becke, Density functional Thermochemistry III. The Role of Exact Exchange, J. Chem. Phys., 1993, 98(7), 5648-5652.

43 T. H. Dunning and P. J. Hay, Gaussian Basis Sets for Molecular Calculations, in Methods of Electronic Structure Theory, Springer US, Boston, MA, 1977, pp. 1-27.

44 J. Tomasi, B. Mennucci and R. Cammi, Quantum Mechanical Solvation Models, Chem. Rev., 2005, 105, 2999-3094.

45 M. J. Frisch, G. W. Trucks, H. B. Schlegel, G. E. Scuseria, M. A. Robb, J. R. Cheeseman, G. Scalmani, V. Barone, G. A. Petersson, H. Nakatsuji, X. Li, M. Caricato, A. Marenich, J. Bloino, B. G. Janesko, R. Gomperts, B. Mennucci, H. P. Hratchian, J. V. Ortiz, A. F. Izmaylov, J. L. Sonnenberg, D. Williams-Young, F. Ding, F. Lipparini, F. Egidi, J. Goings, B. Peng, A. Petrone, T. Henderson, D. Ranasinghe, V. G. Zakrzewski, J. Gao, N. Rega, G. Zheng, W. Liang, M. Hada, M. Ehara, K. Toyota, R. Fukuda, J. Hasegawa, M. Ishida, T. Nakajima, Y. Honda, O. Kitao, H. Nakai, T. Vreven, K. Throssell, J. A. Montgomery Jr, J. E. Peralta, F. Ogliaro, M. Bearpark, J. J. Heyd, E. Brothers, K. N. Kudin, V. N. Staroverov, T. Keith, R. Kobayashi, J. Normand, K. Raghavachari, A. Rendell, J. C. Burant, S. S. Iyengar, J. Tomasi, M. Cossi, J. M. Millam, M. Klene, C. Adamo, R. Cammi, J. W. Ochterski, R. L. Martin, K. Morokuma, O. Farkas, J. B. Foresman and D. J. Fox, Gaussian 09, Revision A.02, Gaussian, Inc., Wallingford CT, 2016.

46 G. Auböck and M. Chergui, Sub-50-Fs Photoinduced Spin Crossover in $\left[\mathrm{Fe}(\mathrm{Bpy})_{3}\right]^{2+}$, Nat. Chem., 2015, 7(8), 629-633.

47 W. Zhang and K. J. Gaffney, Mechanistic Studies of Photoinduced Spin Crossover and Electron Transfer in Inorganic Complexes, Acc. Chem. Res., 2015, 48, 22.

48 A. S. Del Negro, C. J. Seliskar, W. R. Heineman, S. E. Hightower, S. A. Bryan and B. P. Sullivan, Highly Oxidizing Excited States of Re and Tc Complexes, J. Am. Chem. Soc., 2006, 128(51), 16494-16495. 
49 S. Paulson, B. P. Sullivan and J. V. Caspar, Luminescent Ligand-to-Metal Charge-Transfer Excited States Based on Pentamethylcyclopentadienyl Complexes of Tantalum, J. Am. Chem. Soc., 1992, 6905-6906.

$50 \mathrm{Y}$. F. Lee and J. R. Kirchhoff, Absorption and Luminescence Spectroelectrochemical Characterization of a Highly Luminescent Rhenium(I1) Complex, J. Am. Chem. Soc., 1994, 116(14), 3599-3600. 